\title{
Thermoluminescence properties of undoped diamond films deposited using HF CVD technique
}

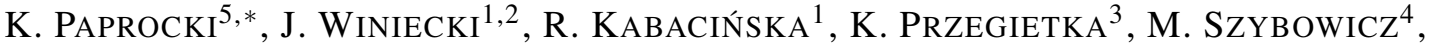 \\ K. FABISIAK ${ }^{5}$ \\ ${ }^{1}$ Oncology Center, Medical Physics Department, Bydgoszcz, Poland \\ ${ }^{2}$ Chair and Clinic Oncology and Brachytherapy, Nicolaus Copernicus University, Toruń, Poland \\ ${ }^{3}$ Institute of Physics, Nicolaus Copernicus University, Torun, Poland \\ ${ }^{4}$ Faculty of Technical Physics, Poznan University of Technology, Piotrowo 3, 60-965 Poznan, Poland \\ ${ }^{5}$ Institute of Physics, Kazimierz Wielki University, Bydgoszcz, Poland
}

\begin{abstract}
Natural diamond has been considered as a perspective material for clinical radiation dosimetry due to its tissuebiocompatibility and chemical inertness. However, the use of natural diamond in radiation dosimetry has been halted by the high market price. The recent progress in the development of CVD techniques for diamond synthesis, offering the capability of growing high quality diamond layers, has renewed the interest in using this material in radiation dosimeters having small geometrical sizes. Polycrystalline CVD diamond films have been proposed as detectors and dosimeters of $\beta$ and $\alpha$ radiation with prospective applications in high-energy photon dosimetry. In this work, we present a study on the TL properties of undoped diamond film samples grown by the hot filament CVD (HF CVD) method and exposed to $\beta$ and $\alpha$ radiation. The glow curves for both types of radiation show similar character and can be decomposed into three components. The dominant TL peaks are centered at around $610 \mathrm{~K}$ and exhibit activation energy of the order of $0.90 \mathrm{eV}$.
\end{abstract}

Keywords: HF CVD undoped diamond; thermoluminescence; glow curve deconvolution; Raman scattering

\section{Introduction}

The diamond is characterized by exceptional mechanical, thermal, optical properties and excellent electrical properties such as a high carrier mobility and high breakdown voltage $[1,2]$. Nowadays, due to the fast development of diamond synthesis from vapor phase, growing diamonds of desired quality and reproducibility became possible. Due to the excellent properties, especially large energy gap, diamond is a very good material for different applications [3, 4]. It can be considered as an excellent material for detection of any kind of radiation [5] and especially can be applied in thermoluminescence (TL) dosimetry [6, 7].

The TL studies of this material allow one to characterize the properties of defects (traps) responsible for energy accumulation of ionizing

\footnotetext{
*E-mail: paprocki@ukw.edu.pl
}

radiation. This phenomenon is vital for passive luminescence (TL or OSL) dosimeters. However, in the case of isolators applied as active detectors, such traps play a harmful role as they are responsible for delay and distortion of the detector response.

TL efficiency of CVD diamond layers depends on many factors including deposition parameters of diamond growth process, i.e. crystalline quality, but also on the type and doping level [8]. Diamond films may be characterized by very distinctive morphological and structural defects, including charge trapping defects responsible for the thermally stimulated radiative recombination. Properties of such metastable localized states for the TL phenomenon obviously have a big influence on the quality of the dosimeter made from diamond film [9-15].

In particular, the optimization of TL properties can be also done by doping of diamond layers by boron or nitrogen incorporated into the CVD 
precursor gas during diamond growth process. The diamond doping with boron or nitrogen is currently a subject of intensive research. The investigation is focused on improving the efficiency of TL sensitivity and TL signal-to-dose linearity $[6,7,16]$. It is well known that the TL signal of undoped CVD diamond films strongly depends on the CVD diamond growth process i.e. precursor gas components and their relative concentrations, substrate temperature, and other technical parameter, for example the filament-substrate distance, gas flow rate and total pressure in the reactor during film deposition [16]. Due to the large number of technological parameters, the TL efficiency of CVD diamond layers needs to be still further investigated.

In this work, we present preliminary results of studying the TL properties of undoped CVD diamond films exposed to $\beta$ and $\alpha$ radiations in relation to their structural quality studied by SEM and Raman spectroscopy. Our preliminary results show that CVD diamond films can be used in the future as accurate detectors of ionizing radiation. The diamonds are considered as tissue equivalent, which makes them particularly suitable for medical applications i.e. brachytherapy and Intraoperative Radiation Therapy IORT.

\section{Experimental}

The polycrystalline diamond films with a thicknesses of about $1.5 \mu \mathrm{m}$ were deposited on a $\left(\begin{array}{lll}1 & 0 & 0\end{array}\right)$ silicon substrate by the Hot Filament Chemical Vapor Deposition (HF CVD) technique. A mixture of methanol and hydrogen $\left(\mathrm{CH}_{3} \mathrm{OH} / \mathrm{H}_{2}=1 \%\right)$ was used as a working gas. The parameters of the growth process were as follows: the total pressure in the reaction chamber: $\mathrm{p}=8 \mathrm{kPa}$, the substrate temperature: $1100 \mathrm{~K}$ and the working gas flow rate: $100 \mathrm{sccm}$ (standard cubic centimeter per minute). In order to enhance the diamond nucleation density, the silicon substrate before the deposition process, was scratched with a diamond paste [17].

The Raman spectrum was recorded, at room temperature in air, in the backscattering geometry. The blue line $(488 \mathrm{~nm})$ from an argon laser was used as the excitation source.
The TL experiments were carried out with help of RISO TL/OSL-DA-20 luminescence reader [18], equipped with EMI9235QA photomultiplier and beta and alpha radiation sources. Such equipment is commonly used in the field of luminescence dating [19], but it can be also applied for TL measurements in material studies [20]. The sample was put on a stainless steel disc $(9.7 \mathrm{~mm}$ in diameter) and silicon oil was used as an adhesive. The sample was kept and operated in light tight conditions. Prior to the irradiation it was preheated up to $450{ }^{\circ} \mathrm{C}$.

The TL was measured without any optical emission filters at a heating rate of $1 \mathrm{~K} / \mathrm{s}$ in argon atmosphere. TL analysis was carried out using GlowFit program for deconvolution of glow curves, described in detail in the literature [21], from which the kinetic parameters were extracted.

The nominal dose of beta radiation of $940 \mathrm{~Gy}$ applied from ${ }^{90} \mathrm{Sr} /{ }^{90} \mathrm{Y}$ source in a standard arrangement was estimated on the basis of the dose rate, as calibrated for quartz grains with the diameter of the order of 200 micrometers [22]. The alpha irradiation was realized using incorporated ${ }^{241} \mathrm{Am}$ foil source of nominal activity of $10.7 \mathrm{MBq}$ under vacuum conditions (the chamber was evacuated below $10 \mathrm{kPa}$ ). The $27 \mathrm{~Gy}$ of the nominal dose of alpha radiation was calculated assuming $45 \mathrm{mGy} / \mathrm{s}$ of the dose rate, as expected for fine quartz grains with the diameter of 10 micrometers. However, the actual dose absorbed by the sample as a result of irradiation is governed by charge build-up, attenuation and backscattering. For a given radiation type and energy, the charge build-up and attenuation depend on the thickness (grain size) of the sample and its composition, while backscattering is determined mainly by the sample carrier. In case of quartz grains used in calibration, the backscattering is influenced by the disc but in case of thin diamond layer deposited on Si wafer, the backscattering is affected mainly by $\mathrm{Si}$ substrate. Therefore, nominal doses estimated on the basis of dose rates, calibrated for quartz grains, should not be treated as true absolute values for diamond samples, but rather as relative doses corresponding to a given sample and particular radiation [22]. 


\section{Results and discussion}

The Raman spectroscopy is a fingerprint of each crystal structure, therefore it is an ideal and nondestructive tool for detection of different forms of crystalline and amorphous carbons, characterized by $\mathrm{sp}^{2}$ and $\mathrm{sp}^{3}$ hybridized $\mathrm{C}-\mathrm{C}$ bonds [23] and for this reason it is also frequently used for the analysis of CVD diamond quality.

The phonon spectrum of diamond crystal is characterized by triply degenerated phonon mode in the center of Brillouin zone which gives a single, sharp peak at $1332 \mathrm{~cm}^{-1}$ in the Raman spectrum, associated with the first-order phonon mode.

The SEM photo and Raman spectrum of the diamond sample are shown in Fig. 1.
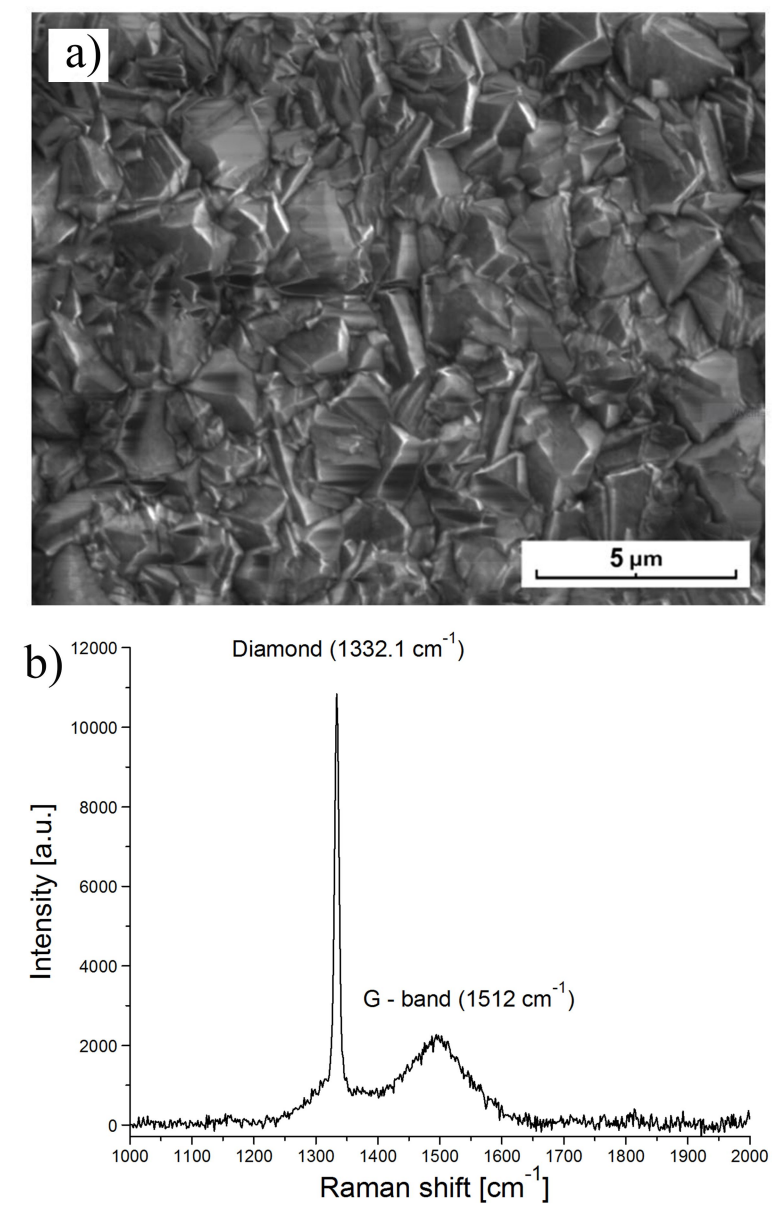

Fig. 1. HF CVD undoped diamond film before irradiation: (a) SEM photo, (b) Raman spectrum (after PL background subtraction).
The recorded Raman spectrum indicates that our diamond film is of relatively high quality as it is indicated by FWHM (full width at half maximum ) which is equal to $4.3 \mathrm{~cm}^{-1}$. The Raman spectrum shows also a low intensity broad band peaked at around $1510 \mathrm{~cm}^{-1}$ (G-band) indicating that there may be a small amount of graphitic or amorphous carbon phase present in the film.

TL glow curves after subtraction of the background (originating from incandescence of heating strip, i.e. black body radiation) are presented in Fig. 2.

The TL curves were deconvoluted using GlowFit software assuming three components of the first order kinetics.

As a result, the values for the activation energy $\mathrm{E}(\mathrm{eV})$ and attempts-to-escape frequency $\mathrm{s}\left(\mathrm{s}^{-1}\right)$ for every component have been obtained (Table 1 and Table 2).

As it can be noticed from Fig. 2, both glow curves recorded after $\alpha$ and after $\beta$ irradiations show similar shapes. Deconvolution revealed two dominant TL peaks in a high-temperature region: around $600 \mathrm{~K}$ and $500 \mathrm{~K}$ and less intense lowtemperature peak near $400 \mathrm{~K}$.

One of the most popular methods for analyzing TL glow curves is the method proposed by Chen [24] and Pimpalshende et al. [25]. Fig. 3 presents the idea of this method.

Depending on a shape of the glow curve, the activation energy can be calculated from the following equation $[25,26]$ :

$$
E_{\lambda}=C_{\lambda}\left(k T_{m}^{2} / \lambda\right)-b_{\lambda}\left(2 k T_{m}\right)
$$

where $\lambda$ is $\tau, \delta$ or $\omega$ and $C_{\lambda}$ and $b_{\lambda}$ are given by:

$C_{\tau}=1.51+3(\mu-0.42)$ and $b_{\tau}=1.58+4.2(\mu-0.42)$

$$
\begin{aligned}
& C_{\delta}=0.976+7.3(\mu-0.42) \text { and } b_{\delta}=0 \\
& C_{\omega}=2.52+10.2(\mu-0.42) \text { and } b_{\omega}=1
\end{aligned}
$$

The value of $\mu\left(\mu=\left(T_{2}-T_{m}\right) /\left(T_{2}-T_{1}\right)\right.$ is 0.42 for first-order kinetics and 0.52 for 

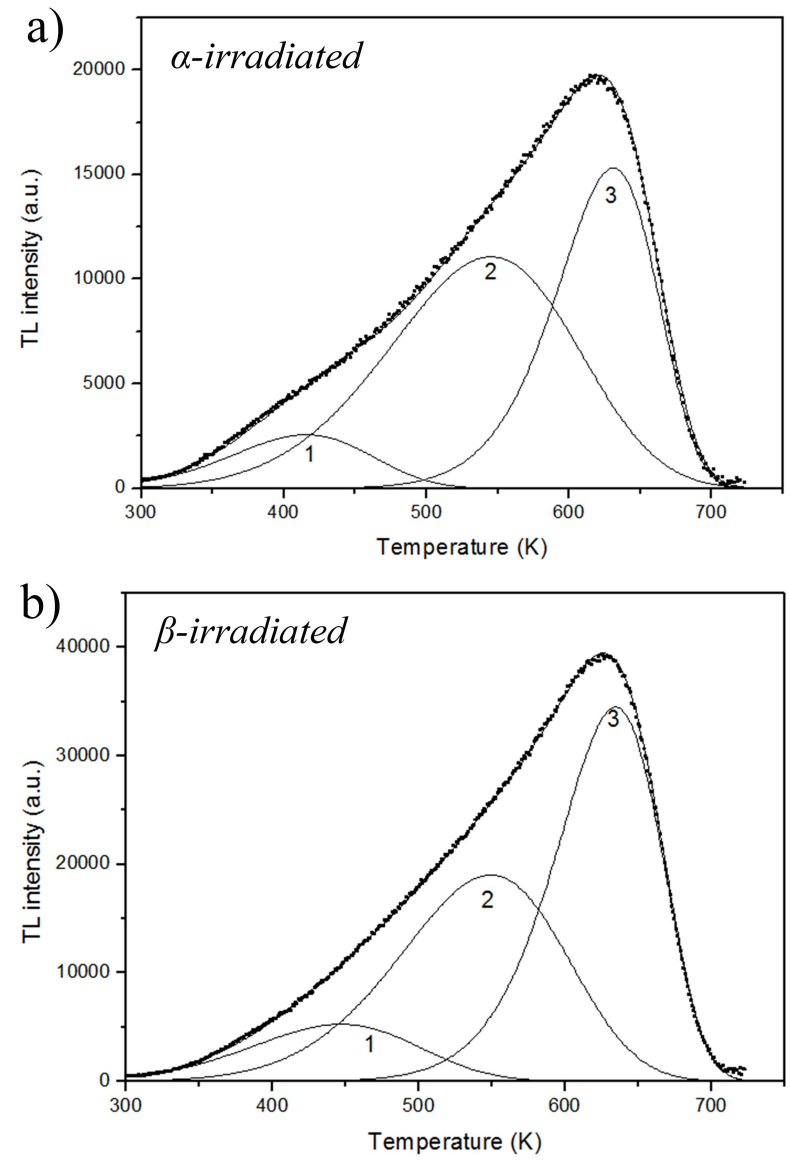

Fig. 2. TL glow curves and fitting results for the sample of polycrystalline CVD diamond films irradiated with (a) $\alpha$ nominal dose of $27 \mathrm{~Gy}$ and (b) $\beta$ nominal dose of $940 \mathrm{~Gy}$.

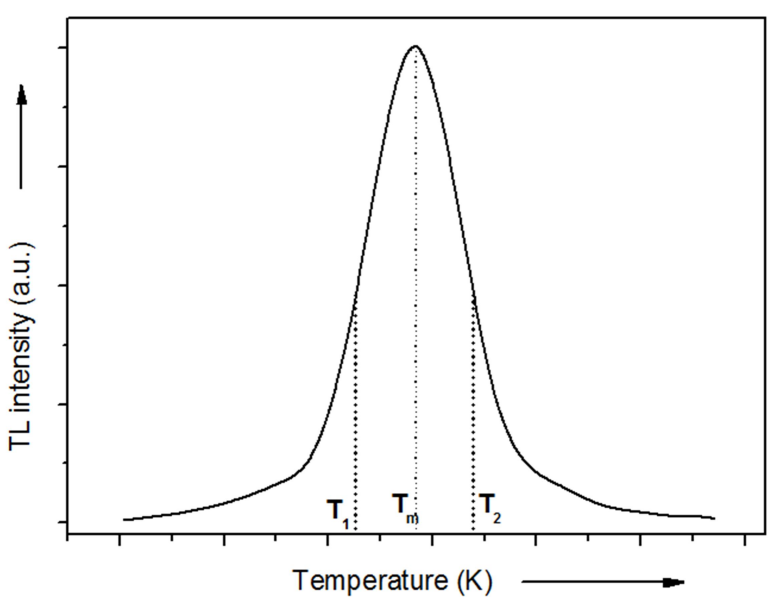

Fig. 3. The idea of the method for glow curve shape analysis. second-order kinetics [25]. The value of $\rho$ $\left(\rho=\left(T_{2}-T_{m}\right) /\left(T_{m}-T_{1}\right)\right.$ ranges from 0.7 to 0.9 for first-order kinetics and from 1.05 to 1.2 for second-order kinetics.

The TL glow curve parameters for CVD diamond film are collected in Table 1 and Table 2.

From Fig. 2 it follows that although $\beta$ nominal dose is 35 times higher in comparison to $\alpha$ nominal dose, the ratio of TL intensity excited by $\beta$ irradiation to TL intensity excited by $\alpha$ irradiation is only of the order of 3. Surprisingly, low TL sensitivity to $\beta$ radiation can be explained by extremely low thickness of the diamond layer.

Let us consider this problem in details. Strontium-90 has a half-life of 28.8 years and average energy of emitted beta-particles (betas) is only $0.196 \mathrm{MeV}$. The daughter nucleus yttrium-90 has a half-life of $64 \mathrm{~h}$ and emits betas with an average energy of $0.927 \mathrm{MeV}$. In the source, both radioisotopes are in equilibrium and both $\beta$ components contribute to the $\beta$ dose. The values of maximum depth of penetration were estimated as $90 \%$ of CSDA range read from NIST database [27]. The maximum ranges of betas in diamond are: $130 \mathrm{mi}-$ crons and 1100 microns for $0.2 \mathrm{MeV}$ and $0.9 \mathrm{MeV}$, respectively. Hence, the thickness of the diamond layer (c.a. 1.5 microns) is much less than the average range of $\beta$ particles from Sr-90/Y-90 source.

In such short distance, the energy transfer from $\beta$ particles to the absorber is less effective due to build-up phenomenon, which is even more important for low energy electrons. The depth required to develop build-up is of the order of $1 / 3$ to $1 / 2$ of the maximum range and it is not satisfied by the thickness of our diamond sample. Because of that, actual dose deposited in the CVD is much lower than the value estimated from the dose rate calibrated for bigger quartz grains, whose size assures build up conditions [22].

Regarding the alpha particles emitted from Am241 source (main energy range 5.5 to $5.6 \mathrm{MeV}$ ), their maximum depth estimated as $99 \%$ of CSDA range read from NIST database [27] appears to be of the order of 15 microns. It is still more than the sample thickness and one has to keep in mind that 
Table 1. TL glow curve parameters for CVD diamond film after $\beta$-irradiation.

\begin{tabular}{cccccccccc}
\hline Band & $\begin{array}{c}\mathrm{Tm} \\
{[\mathrm{K}]}\end{array}$ & $\begin{array}{c}\mathrm{E} \\
{[\mathrm{eV}]}\end{array}$ & $\begin{array}{c}\mathrm{s} \\
{[1 / \mathrm{s}]}\end{array}$ & $\begin{array}{c}\mathrm{T}_{1} \\
{[\mathrm{~K}]}\end{array}$ & $\begin{array}{c}\mathrm{T}_{2} \\
{[\mathrm{~K}]}\end{array}$ & $\mu$ & $\begin{array}{c}\mathrm{E}_{\mathcal{\tau}} \\
{[\mathrm{eV}]}\end{array}$ & $\begin{array}{c}\text { Ballarin } \\
\text { factor } \rho\end{array}$ & $\begin{array}{c}\text { Kinetic } \\
\text { order }\end{array}$ \\
\hline \hline 1 & 429 & 0.2529 & $1.40 \cdot 10^{3}$ & 359 & 490 & 0.47 & 0.29 & 0.87 & Mixed \\
2 & 526 & 0.4316 & $1.35 \cdot 10^{4}$ & 460 & 580 & 0.45 & 0.47 & 0.82 & 1 \\
3 & 610 & 0.8336 & $2.07 \cdot 10^{8}$ & 561 & 648 & 0.44 & 0.89 & 0.78 & 1 \\
\hline
\end{tabular}

Table 2. TL glow curve parameters for CVD diamond film after $\alpha$-irradiation.

\begin{tabular}{cccccccccc}
\hline Band & $\begin{array}{c}\mathrm{Tm} \\
{[\mathrm{K}]}\end{array}$ & $\begin{array}{c}\mathrm{E} \\
{[\mathrm{eV}]}\end{array}$ & $\begin{array}{c}\mathrm{s} \\
{[1 / \mathrm{s}]}\end{array}$ & $\begin{array}{c}\mathrm{T}_{1} \\
{[\mathrm{~K}]}\end{array}$ & $\begin{array}{c}\mathrm{T}_{2} \\
{[\mathrm{~K}]}\end{array}$ & $\mu$ & $\begin{array}{c}\mathrm{E}_{\tau} \\
{[\mathrm{eV}]}\end{array}$ & $\begin{array}{c}\text { Ballarin } \\
\text { factor } \rho\end{array}$ & $\begin{array}{c}\text { Kinetic } \\
\text { order }\end{array}$ \\
\hline \hline 1 & 388 & 0.2912 & $2.08 \cdot 10^{3}$ & 336 & 431 & 0.49 & 0.34 & 0.94 & Mixed \\
2 & 514 & 0.3086 & $3.41 \cdot 10^{3}$ & 431 & 585 & 0.46 & 0.35 & 0.86 & 1 \\
3 & 603 & 0.8612 & $4.28 \cdot 10^{8}$ & 556 & 639 & 0.43 & 0.90 & 0.77 & 1 \\
\hline
\end{tabular}

alpha particles deposit most of their energy in the final length of their path in the absorber. Therefore, the nominal alpha dose of $27 \mathrm{~Gy}$ is surely overestimation of the actual dose absorbed by the sample.

The performed TL analysis gives indications and a great deal of information relative to defects present in the films and may be a valuable complementary tool for characterizing polycrystalline CVD diamond films.

Up to now, studies on CVD diamond as TLD material have tended to focus on TL response as a function of doping $[7,16]$. The comparison between undoped and N-doped CVD diamond do not show any clear trends in TL yield. Although doping levels due to inclusion of $\mathrm{N}$ may have contributed to TL response, direct comparison with undoped CVD diamond is difficult due intrinsic defect levels present in all CVD diamond.

\section{Conclusions}

The TL sensitivity of investigated sample appears to be very low due to very small thickness of the film. As a matter of fact, only small fraction of radiation dose is delivered into the CVD film. Most of $\alpha$ and $\beta$ particles pass through the diamond layer and they are stopped in Si substrate, where the major part of radiation dose is imparted. Additionally, the low TL sensitivity can also be attributed to low concentration of defects connected with TL traps. Three types of traps responsible for TL signal can be recognized from glow curve deconvolution assuming first order TL kinetics. The glow curves of the investigated sample exhibit one dominant peak with maximum around $610 \mathrm{~K}$. It is connected with traps with the activation energy $\mathrm{E}$ of the order of $0.85 \mathrm{eV}$ and frequency factor $\mathrm{s}$ of the order of $3 \cdot 10^{8} \mathrm{~s}^{-1}$. However, for the shallower traps, their extremely low values of frequency factor s suggest that their kinetic order can be higher than 1 and possibly many other shallow traps (continuously distributed) can be responsible for TL in a low temperature region.

More studies are needed to determine dosimetric features of the sample as well as reproducibility and stability of TL response.

\section{Acknowledgements}

TL measurements were performed with help of infrastructure of the Centre for Modern Interdisciplinary Technologies, the Nicolaus Copernicus University in Torun, ul. Wilenska 4, 87-100 Torun, Poland.

\section{References}

[1] Spear K.E., Dismukes J.P., Synthetic diamond: emerging CVD science and technology, John Wiley \& Sons, New York, 1994.

[2] PAN L., Kania D., Science, 255 (1992), 830.

[3] Piliero M., Hugtenburg R., Ryde S., Oliver K., Radiat. Phys. Chem., 104 (2014), 10.

[4] Zakari Y., Mavunda R., NAm T., Keddy R., Physcs. Proc., 37 (2012), 950. 
[5] Lansley S.P., Gaudin O., Whitfield M.D., McKeag R.D., Rizvi N., JackMan R.B., Diam. Relat. Mater., 9 (2000), 195.

[6] Benabdesselam M., Iacconi P., Butler J., BRIAND D., Diam. Relat. Mater., 10 (2001), 2084.

[7] Descamps C., Tromson D., Guerrero M., Mer C., Rzepka E., Nesladek M., Bergonzo P., Diam. Relat. Mater., 15 (2006), 833.

[8] Nebel C., Semicond. Sci. Tech., 18 (2003), S1.

[9] Vittone E., Manfredotti C., Fizzotti F., GiuDice A.L., Polesello P., Ralchenko V., Diam. Relat. Mater., 8 (1999), 1234.

[10] Borchi E., Furetta C., Kitis G., Leroy C., Sussmann R., Whitehead A., Radiat. Prot. Dosim., 65 (1996), 291.

[11] Benabdesselam M., Iacconi P., Briand D., Lapraz D., Butler J., Radiat. Prot. Dosim., 84 (1999), 257.

[12] Marczewska B., Olko P., Nesladek M., Waligorski M., Kerremans Y., Radiat. Prot. Dosim., 101 (2002), 485

[13] Bogani F., Borchi E., Bruzzi M., Leroy C., SCIORTINO S., Nucl. Instrum. Meth. A, 388 (1997), 427.

[14] Rębisz M., Guerrero M., Tromson D., PoMORSKI M., MARCZEWSKA B., NesladeK M., Bergonzo P., Diam. Relat. Mater., 13 (2004), 796.

[15] Marczewska B., Bilski P., NesladeK M., Olko P., RęBisz M., WaligóRski M.P.R., Phys. Status Solidi A, 193 (2002), 470.

[16] Barboza-Flores M., Gastélum S., CruZZaragoza E., Meléndrez R., Chernov V., Pedroza-Montero M., Favalli A., Radiat. Meas., 43 (2008), 379.
[17] Fabisiak K., BaŁa W., Paprocki K., Szreiber M. UNISZKIEWICZ C., Opt. Mater., 31 (2009), 1873.

[18] Thomsen K.J., BøtTer-Jensen L., Denby P.M., Moska P., Murray A., Radiat. Meas., 41 (2006), 768.

[19] OczKowski H.L., PrZegietka K., Radiat. Meas., 29 (1998), 435.

[20] ZAŁEsSKI M., Borowiec M., SZYMCZAK H., PrZegiętKa K., OCZKOWSKi H., Opt. Commun., 151 (1998), 46.

[21] Puchalska M., Bilski P., Radiat. Meas., 41 (2006), 659.

[22] Przegietka K., Chruscinska A., Radiat. Phys. Chem., 104 (2014), 118.

[23] Meléndrez R., Chernov V., May P., CAStaneda B., Pedroza-Montero M., BarbozaFlores M., Phys. Status Solidi A, 206 (2009), 2103.

[24] Chen R., J. Appl. Phys., 40 (1969), 570.

[25] Pimpalshende D., Dhoble S., Luminescence, 29 (2014), 1019.

[26] Pimpalshende D., Dhoble S., Luminescence, 30 (2015), 144.

[27] Berger M., Coursey J., Zucker M., Chang J., National Institute of Standards and Technology, 2005.

Received 2017-02-09

Accepted 2017-11-21 